\title{
OS EFEITOS DA HIDROTERAPIA NO PÓS-OPERATORIO DO LIGAMENTO \\ CRUZADO ANTERIOR (LCA)
}

\section{HE EFFECTS OF HYDROTHERAPY IN THE POST-OPERATION OF THE PREVIOUS CROSS LINK (ACL)}

Joice Dias Meireles

Acadêmica do $9^{\circ}$ período do Curso de Fisioterapia da -UNIPAC.

joicediasmeireles@gmail.com.

Matteus Cordeiro de Sá

Pós-Graduado em Fisioterapia Traumato Ortopédica -UNIPAC. Curso de Fisioterapia matteuscordeirodesa@gmail.com

Raiane Vieira da Silva

Acadêmica do $9^{\circ}$ período do Curso de Fisioterapia da -UNIPAC.

Carlos - UNIPAC. E-mail: raianevieira1995@ hotmail.com.

\section{Resumo}

O joelho é uma das articulações mais complexas do corpo humano com grande amplitude de movimentos, possibilitando os movimentos de flexão, extensão, assim como, uma leve rotação lateral e medial, dentre as estruturas presentes nessa articulação o ligamento cruzado anterior se caracteriza por promover estabilidade ao joelho. Os tratamentos das rupturas no ligamento cruzado anterior sofreram diversas alterações ao longo dos anos, desse modo, existem grandes contribuições e vantagens para a reabilitação no pós-operatório, com métodos e técnicas eficazes, sendo possível citar a hidroterapia como uma técnica capaz de viabilizar a melhora e a funcionalidade da área lesionada. Dessa maneira, o presente trabalho buscou relatar os efeitos da hidroterapia em pacientes pós-operados 
de lesões no ligamento cruzado anterior, por meio da discrição das principais ameaças sofridas ao LCA, bem como, o delineamento de como é realizado o tratamento pós-cirúrgico no LCA e a demonstração dos benefícios proporcionados pelo meio aquático, por intermédio, do desenvolvimento de uma pesquisa literária, considerada como qualitativa quanto à abordagem do problema e descritiva quanto aos objetivos de estudo, constatando que a hidroterapia por possibilitar alívio na dor do paciente, além de assegurar a melhora na condição física deste, assim como, na sua capacidade motora, é uma grande aliada no processo de reabilitação.

Palavras-chave: Ligamento Cruzado Anterior; Hidroterapia; Joelho; Pós-operatório.

\begin{abstract}
The knee is one of the most complex joints of the human body with wide range of motion, allowing the movements of flexion, extension, as well as a slight lateral and medial rotation, among the structures present in this joint the anterior cruciate ligament is characterized by promoting stability to the knee. The treatment of anterior cruciate ligament ruptures has undergone several changes over the years, thus, there are great contributions and advantages for rehabilitation in the postoperative period, with effective methods and techniques, and it is possible to mention hydrotherapy as a technique capable of enabling improvement and functionality of the injured area. Thus, the present study sought to report the effects of hydrotherapy in postoperative patients of lesions in the anterior cruciate ligament, through the discretion of the main threats to ACL, as well as the delineation of how is performed the post-surgical treatment in ACL and the demonstration of benefits provided by the aquatic environment, through, the development of a literary research, considered as qualitative as to the approach to the problem and descriptive as to the study objectives, noting that hydrotherapy for enabling pain relief of the patient, in addition to ensuring the improvement in the physical condition of the patient, as well as in his motor capacity, is a great ally in the rehabilitation process.
\end{abstract}

Keywords: Anterior Cruciate Ligament; Hydrotherapy; Knee; Post-operative.

\title{
1. Introdução
}

O joelho é uma das articulações mais complexas do corpo humano com grande amplitude de movimentos, possibilitando os movimentos de flexão, extensão, assim como, uma leve rotação lateral e medial. Desse modo, a realização do movimento de flexão permite que os meniscos sejam tracionados para trás, já a extensão dá ao joelho uma movimentação 
inversa, sendo esta caracterizada pelo ato de esticar a perna, as rotações medial e lateral acontecem somente com a flexão do joelho, consequentemente, as rotações são fundamentadas pela adução e abdução do pé. (DUTHON et al., 2006).

Devido às ações realizadas pelo joelho, o ato de aproximar ou afastar o membro de sua raiz é de suma importância para o deslocamento do corpo humano, sendo uma articulação intermediária do membro inferior, responsável por suportar grandes esforços, instável do ponto de vista ósseo, onde o sistema ligamentar e muscular são seus principais estabilizadores, diante disso o ligamento cruzado anterior (LCA) sofre com frequência sua ruptura total, geralmente tal lesão é ocasionada com a torção do joelho, no momento em que o pé se encontra fixo no chão. (NETTER, 2008).

Existem grandes contribuições e vantagens para a reabilitação no pós-operatório, com métodos e técnicas eficazes, sendo possível citar a hidroterapia como uma técnica capaz de viabilizar a melhora e a funcionalidade da área lesionada proporcionando ao indivíduo continuidade com suas funções cotidianas, permitindo uma ampla abordagem e atuação necessária para tal patologia.

Os tratamentos das rupturas no ligamento cruzado anterior sofreram diversas alterações ao longo dos anos. A insatisfação com os resultados dos procedimentos realizados no passado e a evolução dos conhecimentos da articulação do joelho permitiu alcançar o estágio atual, onde se consegue uma alta taxa de sucesso com a reconstrução intra-articular.

Partindo desta problemática seria a hidroterapia uma alternativa de reabilitação do paciente no pós-operatório do ligamento cruzado anterior (LCA)?

Dessa maneira, o presente trabalho buscou relatar os efeitos da hidroterapia em pacientes pós-operados de lesões no ligamento cruzado anterior, por meio da discrição das principais ameaças sofridas ao LCA, bem como, o delineamento de como é realizado o tratamento pós-cirúrgico no LCA e a demonstração dos benefícios proporcionados pelo meio aquático. 
Fazendo-se necessário este estudo, uma vez que, a hidroterapia é um meio fisioterapêutico utilizado no que diz respeito a reabilitação e/ou prevenção de variações funcionais, visando resultados fisiológicos, cinesiológicos e físicos.

Com isso, este trabalho foi desenvolvido utilizando o método de pesquisa literária, sendo considerada qualitativa quanto à abordagem do problema e descritiva quanto aos objetivos de estudo. A seleção de tais procedimentos se deu por adequar-se melhor aos objetivos pretendidos, em razão de que a descrição por diversos profissionais auxilia no entendimento do objeto em estudo. Foi realizada uma revisão da literatura científica de revistas, artigos e de obras publicadas em formato físico e também eletrônico indexada em bases de dados como SciELO, através do mecanismo de busca do Google Acadêmico. Optouse por esta base de dados por ser esta uma das principais fontes de publicações científicas, considerando tal procedimento como critério inicial para seleção.

O material literário utilizado nesta obra foi selecionado com base em seus aparentes resultados na utilização da hidroterapia como um recurso terapêutico em pacientes no póscirúrgico. Como critério de seleção buscou-se trabalhos com as seguintes palavras-chave: hidroterapia, LCA, fisioterapia aquática, reconstrução do LCA, entre outras. Considerando que tais trabalhos estão na língua portuguesa ou inglesa e foram publicados no período compreendido entre os anos de 2005 até 2019, tendo em vista que, aqueles que possuíam uma grande relevância histórica perante o tema abordado e não estavam neste período de seleção foram utilizados. Como critério de exclusão, foram eliminados aqueles trabalhos que não tinham teóricos para fundamentar esta obra e por isso não tinham base acadêmica suficiente para embasar uma pesquisa.

\section{O Joelho e o Ligamento Cruzado Anterior (LCA)}

O joelho está presente no sistema osteo-articular de sustentação servindo como peça chave para a locomoção do corpo humano, tal articulação encontra-se localizada entre o fêmur e a tíbia, inicialmente o joelho era conhecido por ser uma articulação dobradiça, devido 
os seus principais movimentos que são a flexão e extensão, atualmente se tem o conhecimento de que o joelho além dos seus principais movimentos realiza uma rotação longitudinal sobre a perna, sendo esta caraterizada como uma movimentação acessória. (TRILHA et al., 2009).

Diante de todas as articulações presentes no corpo humano, a articulação do joelho se caracterizar por ser a maior e mais complexa, que mesmo apresentando uma única cavidade articular o joelho se divide em três articulações interligadas, sendo elas a articulação intermediária, a articulação lateral e a articulação medial. (MARIEB; HOEHN, 2009).

É notório que as principais estruturas presentes no joelho são a cápsula articular, o ligamento da patela, o ligamento poplíteo oblíquo e o arqueado, assim como, o ligamento colateral tibial, o ligamento colateral fibular, o ligamento cruzado anterior, o ligamento cruzado posterior, os meniscos e as bolsas. (TORTORA; DERRICKSON, 2016).

Dentre as estruturas presentes no joelho o ligamento cruzado anterior se caracteriza por promover estabilidade ao joelho, tendo como função a restrição da translação da tíbia relativamente ao fêmur, possuindo outras funções secundárias como a delimitação da rotação interna e externa da tíbia e a angulação em valgo e varo do joelho. (FANELLI, 2012).

Outra particularidade do ligamento cruzado anterior é que ele consegue reter até $75 \%$ da carga translacional anterior, no momento que sua extensão encontra-se completa, essa porcentagem aumenta quando a articulação está em flexão de $30^{\circ}$ a $90^{\circ}$ podendo absorver até 85\% da carga aplicada. (MARKATOS et al., 2013).

No que diz respeito à sua histologia o LCA é composto por partes de resistente tecido fibroso e flexível, possui colágeno em sua maioria do tipo 1, assim como, fibroblastos e fibras elásticas. A estrutura do LCA é análoga com as dos tendões contendo partículas de colágeno arranjadas em fibrilas, que agrupam-se em fibras do mesmo material e formam feixes. (CARNEIRO; JUNQUEIRA, 2008).

\section{As Causas de Lesões no Ligamento Cruzado Anterior (LCA)}

A elevada quantidade de lesões no ligamento cruzado anterior é observada a cada dia 
com mais frequência, devido, tanto à pratica de esportes quanto à recreacional. A literatura demonstra que as lesões estão presentes estatisticamente em $0,30 / 1000$ dos habitantes que praticam esportes. (PRODROMOS et al., 2007).

As causas para lesões no LCA podem ser de contato ou de sobre uso, as lesões de contato são ocasionadas pela pressão existente no determinado instante que supera os limites de resistência do tecido, já as lesões de sobre uso tem sua origem no stress derivado do ato de repetição de um determinado movimento. (SOARES, 2007).

A complexidade existente no que diz respeito em relação à interação dos fatores que causam as lesões no LCA torna mais complexo o estabelecimento de todos os potenciais de risco de lesões no ligamento cruzado anterior. Diante disso, se estabelece que os fatores internos estejam intrínsecos com o indivíduo e os fatores externos estão envolvidos com o ambiente. (IRELAND, 1999).

Dentre os fatores externos é possível verificar sua maior influência em pessoas praticantes de algum esporte podendo ser citado o futebol, onde o nível competitivo aumenta consideravelmente a exposição a lesões no LCA, uma vez que, em disputas de alto nível é claramente percebido que o desempenho do atleta deve ser maior, outro fator externo que se deve destacar é a quantidade de partidas ou competições em que o atleta participou, tendo em vista que quanto maior essa quantidade de partidas, por mais vezes o competidor estará exposto a tal lesão. (ROI et al., 2010).

Em relação aos fatores internos é possível verificar que a idade tem uma enorme influência nas lesões relacionas ao LCA, afinal, quanto mais velho o indivíduo mais tempo de exposição a lesões no ligamento cruzado anterior ele terá, a idade também influencia em relação aos tipos de lesões, observando que em crianças é mais comum a retirada da espinha da tíbia do que a ruptura do ligamento, esta direção das lesões derivadas pela idade se dá ao fato de que na juventude o LCA é mais resistente e em adultos ele é mais flexível. (NORONHA, 2006).

\section{A Hidroterapia como Alternativa de Recuperação após Procedimento no LCA}


No ramo da fisioterapia existem as mais variadas técnicas voltadas para a reabilitação do paciente, dentre elas a hidroterapia ou fisioterapia aquática vem ganhando destaque devido os seus benefícios, incluindo o baixo custo de sua aplicação, sendo executada em piscinas específicas, contendo uma temperatura ideal para cada patologia em que se destina trabalhar. (ARLIANI et al., 2012).

A hidroterapia possui como finalidade a prevenção, assim como, o tratamento no alívio de dores, buscando auxiliar no pós-operatório, bem como doenças específicas, por intermédio da combinação de procedimentos aquáticos, peculiaridades físicas e competências fisioterapêuticas fazendo com que a hidroterapia seja efetiva. (CATTELAN; BORBA; PETROCHI, 2009).

É notório que em pacientes com lesões no LCA, o corpo do paciente potencializa uma predisposição ao atrofiamento do quadríceps, derivada da dor, perda ou edema da amplitude de movimento, uma vez que, a inflamação derivada a partir da lesão produz uma dor constante e intensa, levando a perda temporária dos movimentos do joelho. (XAVIER, 2014).

Mediante a unicidade da água, o empuxo é capaz de aliviar as dores causadas pelo estresse oriundo das articulações sustentadoras de peso permitindo a realização de movimentos com uma quantidade de força gravitacional restaurando a mobilidade do joelho, fazendo com que os movimentos que não suportam pesos sejam executados dentro da água sem dificuldade. (SOARES, 2011).

No pós-operatório do LCA a hidroterapia consegue efetivamente diminuir as dores, restaurar a função do ligamento e aumentar o controle muscular, possibilitando ao paciente a retomada dos níveis de funcionamento prévio ou melhorado, desse modo, existe vários benefícios no processo do pós-operatório com o uso da hidroterapia. (FERNANDES; MACEDO, 2009).

Durante o tratamento com a hidroterapia é possível verificar a percepção de liberdade na movimentação do paciente no ato de andar, ocasionando uma alteração na exigência muscular favorecendo no aumento de massa muscular, possibilitando a melhora no quadro 
clinico, bem como a locomoção e qualidade de vida do pós-operado. (PEREIRA; SOUZA, 2012).

\section{As aplicações da Hidroterapia no Pós-operatório do Ligamento Cruzado Anterior (LCA)}

Faz-se importante a observação dos protocolos de reabilitação, haja vista que, é possível encontrar protocolos de metodologias mais conservadoras a protocolos mais acelerados e intensivos (MARTINI et al., 2016). Percebe-se tal ocorrência quando se verifica a existência de protocolo simplificado com indicação de hidroterapia período de 45 a 90 dias após o procedimento cirúrgico, bem como, existe protocolos que indicam que a hidroterapia pode ser iniciada com 21 dias compreendo até os 90 dias após a cirurgia. (PIMENTA et al., 2012).

Porém, é possível averiguar que mediante as características das lesões ocorridas no LCA, no processo de reabilitação procura-se a existência de uma melhora na amplitude dos movimentos, assim como, na força do músculo, um ganho de equilíbrio, além da diminuição do edema e da dor. Desse modo, é comum a prática de treinamentos proprioceptivos, a crioterapia, a eletroterapia e exercícios de cadeia cinética fechada, como modalidades de reabilitação. (JUNIOR et al., 2009).

Em contrapartida, a hidroterapia vem se destacando em relação aos métodos tradicionais, devido a sua capacidade de permitir uma movimentação ativa e antecipada, bem como a sua capacidade de desenvolver um desempenho neuromuscular. (CATTELAN; BORBA; PETROCINI, 2009).

Para a recuperação do pós-cirúrgico a hidroterapia é uma grande aliada, por possibilitar o alívio na dor do paciente, proporcionando uma boa aceitação de sua parte, viabilizando a melhora na condição física e motora do pós-operado, além de reduzir sua gordura corporal. (SOARES, 2011). 
Com a realização de um estudo literário que em comparação com outras técnicas, foi possível verificar que a hidroterapia demonstra ser mais eficaz, por poder ser utilizada mesmo quando o paciente está sentindo dor, bem como, inflamação e espasmos musculares, conseguindo proporcionar ao paciente um ambiente estável. (KOURI, 2000).

No entanto, após realizar uma revisão de 19 trabalhos direcionados aos efeitos da hidroterapia em relação aos métodos convencionais, verificou que atividades de reabilitação executadas em solo ou no meio aquático não apresentam diferença estatisticamente significativa, mesmo que no meio aquático a musculatura apresente relaxamento, melhora na dor e redução do edema. (Hall et al., 2008).

Assim como, é visto após um estudo de comparação entre os pacientes que realizavam a reabilitação por meio aquático, com pacientes que usufruíam da cinesioterapia em solo, concluiu-se que em ambos os procedimentos os pacientes apresentaram melhoria no seu quadro clínico, demonstrando que o uso da terapia no meio aquático como método de reabilitação teve melhores resultados estatísticos apenas em relação à flexão do joelho.

Ainda no ponto de vista dos autores, em relação à função, a hidrocinesioterapia se mostra superior à cinesioterapia em solo, tal benefício para o autor se dá pela turbulência gerada pela água em um ambiente de instabilidade proporcionando relaxamento muscular, desse modo, diminuindo a dor e o edema com mais agilidade. (ANDRADE, 2008).

Através do acompanhamento de 22 atletas certificou que a hidroterapia é totalmente eficaz perante aos métodos clássicos de reabilitação, visto que ela possibilita uma diminuição na sustentação de peso, controle de edema, bem como, uma resistência constante, diminuição da dor e consequentemente melhora da mobilidade articular. Podendo a hidroterapia certamente ser recomendada para o processo de reabilitação desde a fase inicial. (RAHMANN; BRAUER, 2009).

No acompanhamento de um paciente do sexo masculino, com idade de 36 anos, onde inicialmente este apresentava grau 4 para extensão e 3 para flexão no teste de força muscular com $50^{\circ}$ para extensão e $60^{\circ}$ para flexão nos testes de goniometria. Após as seções de hidroterapia o paciente demonstrou melhorar significativamente em relação a sua amplitude 
de movimento, onde ouve um ganho de $30^{\circ}$ em flexão e $40^{\circ}$ em extensão no joelho lesionado, mensurado com um goniômetro. Quando reavaliado a força muscular, observou-se de 4 em flexão e de 5 para extensão, avaliado de acordo com a escala de Oxford. Foi possível ainda, observar a diminuição do edema que passou de $46 \mathrm{~cm}$ para $42 \mathrm{~cm}$, avaliando através de perimetria. (COELHO, 2018).

Em outro estudo foi possível obter respostas positivas com a hidroterapia mostrando mais uma vez a sua eficácia, após realizar o acompanhamento de uma paciente com idade de 23 anos apresentando ruptura total do LCA, que foi submetida a uma cirurgia de reconstrução do ligamento, sendo válido ressaltar que a paciente não era portadora de qualquer distúrbio neurológico, metabólico, cardiovascular e ortopédico grave, ao se concluir 10 sessões de hidroterapia a paciente demonstrou um aumento da amplitude de movimento, ganho de força e resistência muscular. (CANHOTO et al., 2010).

Existe ainda a associação da hidroterapia com a fisioterapia tradicional, possuindo um excelente resultado além de ser benéfico ao paciente, dado que, com a junção de tais procedimentos faz com que o processo seja mais tolerante e menos doloroso, além de garantir um retorno precoce a sua atividade. Se confirma essa possibilidade após a verificação da combinação da hidroterapia com a cinesioterapia no solo, em 3 jogadores com idade entre os 20 e 44 anos, que sofreram lesão no LCA e necessitaram de cirurgia. (KIM et al., 2010).

Com uma revisão da literatura compreendida entre os anos de 1979 a 2013, certificouse que é necessário um método produtivo de reabilitação do ligamento cruzando anterior independente da técnica cirúrgica utilizada, no entanto, não se aponta a hidroterapia como o método mais eficiente nesse processo, e sim o treinamento proprioceptivo executado de maneira tradicional, uma vez que este, para os autores já possibilita ao joelho estabilidade suficiente para a realização de atividades diárias e expositivas, por garantir equilíbrio estático e dinâmico da articulação. (SANTOS; MEJIA, 2013).

\section{Considerações Finais}


Confirmou-se que a articulação do joelho é caracterizada por ser a maior e mais complexa do corpo humano, demonstrando que se faz necessário o estudo continuo de soluções para o tratamento de suas lesões, buscando sempre a consonância entre os estudiosos e pesquisadores da área.

A partir desde estudo literário foi possível constatar que a hidroterapia é uma grande aliada no processo de reabilitação, por possibilitar alívio na dor do paciente, além de assegurar a melhora na condição física deste, assim como, na sua capacidade motora, esclarecendo que com as sessões de hidroterapia o paciente poderá se movimentar com mais rapidez do que com os métodos convencionais no solo, permitindo uma mobilidade ativa.

Averiguou-se que a hidroterapia pode ser utilizada com a junção de outras metodologias, tendo em vista que, independente do processo cirúrgico o paciente deve recorrer a um tratamento pós-traumático, assim como, observou-se que existe protocolos que auxiliam no processo de reabilitação. Contudo, novos estudos devem ser realizados buscando a junção ou a comparação da hidroterapia com a fisioterapia em solo, visto que, ambos proporcionam uma reabilitação após cirurgia no LCA.

\section{Referências}

ANDRADE, Dayane Laura Vieira et al. Estudo comparativo entre o tratamento cinesioterápico e hidrocinesioterápico no pós-operatório da reconstrução do ligamento cruzado anterior. Conscientiae Saúde, v. 7, n. 2, p. 191-199, 2008.

ARLIANI, Gustavo Gonçalves et al. Lesão do ligamento cruzado anterior: tratamento e reabilitação. Perspectivas e tendências atuais. Revista Brasileira de Ortopedia, 2012.

CANHOTO, M. et al. Reabilitação aquática em pré e pós-operatório do ligamento cruzado anterior no atleta. VI Encontro Latino Americano de Pós-Graduação Universidade do Vale do Paraíba, 2010. 
CARNEIRO, Junqueira; JUNQUEIRA, L. C. U. Histologia básica: texto, atlas. GuanabarKoogan, 2008.

CATTELAN, A. V.; BORBA, A. K.; PETROCHI, A. D. Fisioterapia aquática na reconstrução do ligamento cruzado anterior: relato de caso. Revista Digital Buenos Aires, v. 14, n. 131, p. 1-1, 2009.

COELHO, Carlos Jhone. Efetividade da Assistência Hidroterapeutica no Paciente em PósOperatório de Ligamento Cruzado Anterior. Revista Científica Multidisciplinar Núcleo do Conhecimento. Ano 03, Ed. 07, Vol. 01, pp. 142-150, Julho de 2018. ISSN:24480959.

DUTHON, V. B. et al. Anatomy of the anterior cruciate ligament. Knee surgery, sports traumatology, arthroscopy, v. 14, n. 3, p. 204-213, 2006.

FANELLI, Gregory C. O joelho lesado do ligamento múltiplo: um guia prático para o manejo. 2ed, Springer, 2012.

FERNANDES, Rafael Ferraz; MACEDO, Christiane de Souza Guerino. Eficácia da fisioterapia na funcionalidade e dor de indivíduos com lesão no joelho submetidos a procedimento cirúrgico. Arquivos de Ciências da Saúde da UNIPAR, v. 13, n. 1, 2009.

HALL, J; SWINKELS, A; BRINDDON, J; MCCABE, CS. Does Aquatic Exercise Relieve Pain in Adults With Neurologic or Musculoskeletal Disease? Systematic Review and MetaAnalyses of Randomized Controlled Trials. Arch Phys Med Rehabil, Estados Unidos da América, 89(5), p.873-883, may, 2008.

IRELAND, Mary Lloyd. Anterior cruciate ligament injury in female athletes: epidemiology. Journal of athletic training, v. 34, n. 2, p. 150, 1999.

Júnior SS, Costa RG, Gonçalves SM, Paizante GO. Tratamento fisioterapêutico após reconstrução do ligamento cruzado anterior utilizando enxerto do tendão patelar [trabalho de conclusão de curso]. Governador Valadares: Universidade Vale do Rio Doce (UNIVALE); 2009. 
KIM, Eunkuk et al. Aquatic versus land-based exercises as early functional rehabilitation for elite athletes with acute lower extremity ligament injury: a pilot study. PM\&R, v. 2, n. 8, p. 703-712, 2010.

KOURY, Joanne M. Programa de fisioterapia aquática: um guia para a reabilitação ortopédica. Manole, 2000.

MARIEB, Elaine N.; HOEHN, Katja. Anatomia e fisiologia. Artmed Editora, 2009.

MARKATOS, K. et al. The anatomy of the ACL and its importance in ACL reconstruction. European Journal of Orthopaedic Surgery \& Traumatology, v. 23, n. 7, p. 747-752, 2013.

MARTINI, Fernando Pelinser et al. Avaliação da dor após a reconstrução artroscópica do ligamento cruzado anterior do joelho: estudo duplo cego randomizado para Sulfato de Magnésio intra-articular. Rev. AMRIGS, v. 60, n. 2, p. 92-96, 2016.

NETTER, Frank H. Netter-Atlas de anatomia humana. Elsevier Brasil, 2008.

Noronha JC. Lesões do ligamento cruzado anterior. In: Espregueira-Mendes J, Pessoa P, editors. O Joelho. Lisboa: Lidel - edições técnicas, lda; p. 147-82, 2006.

PEREIRA, Welton Silva; DE SOUZA, André Luiz Velano. Benefícios da cadeia cinética fechada na reabilitação de pacientes com lesão do ligamento cruzado anterior. Corpus et Scientia, v. 8, n. 1, p. 60-66, 2012.

PIMENTA, Thais et al. Protocolos de Tratamento Fisioterápico após Cirurgia do Ligamento Cruzado Anterior. Acta Biomedica Brasiliensia, v. 3, n. 1, p. 27-34, 2012.

PRODROMOS, Chadwick C. et al. A meta-analysis of the incidence of anterior cruciate ligament rupture as a function of gender, sport and knee injury reduction regimen. 
Arthroscopy: The Journal of Arthroscopic and Related Surgery, v. 23, n. 12, p. 13201325. e6, 2007.

RAHMANN, Ann E.; BRAUER, Sandra G.; NITZ, Jennifer C. A specific inpatient aquatic physiotherapy program improves strength after total hip or knee replacement surgery: a randomized controlled trial. Archives of physical medicine and rehabilitation, v. 90, n. 5, p. 745-755, 2009.

ROI, GS et al. Prevalência de reconstruções do ligamento cruzado anterior em futebolistas profissionais. Ciências do esporte para a saúde. v. 1, n. 3, p. 118-121, 2010. SANTOS, Neila Taynara Castro; MEJIA, Dayana Priscila Maia. Lesão do ligamento cruzado anterior e tratamento fisioterapêutico. 2013.

SOARES, J. O treino do futebolista. Lesões-Nutrição. Porto: Porto Editora, 2007.

SOARES, Matheus et al. INTERVENÇÃO FISIOTERAPÊUTICA NO PÓS-OPERATÓRIO DE LESÕES DO LIGAMENTO CRUZADO ANTERIOR. TEMA-Revista Eletrônica de Ciências (ISSN 2175-9553), v. 11, n. 16, 2011.

TORTORA, Gerard J.; DERRICKSON, Bryan. Corpo Humano-: Fundamentos de Anatomia e Fisiologia. Artmed Editora, 2016.

TRILHA JUNIOR, Marcial et al. Three-dimensional numerical simulation of human knee joint mechanics. Acta Ortopédica Brasileira, v. 17, n. 2, p. 18-23, 2009.

XAVIER, Daniel Salgado, Os benefícios da hidroterapia para o tratamento de doenças Quarta-feira, 16 de abril de 2014. 